\title{
HYPOMETHYLATING AGENTS IN TREATMENT OF MYELODYSPLASTIC SYNDROME
}

\author{
Olha Boyko \\ State Institution «Institute of Blood Pathology and Transfusion Medicineof National Academy of \\ Medical Sciences of Ukraine» \\ 45 General Chuprynka str., Lviv, Ukraine, 79044 \\ olyaboyko1411@gmail.com
}

\begin{abstract}
Thelkey to the successful treatment of myelodysplastic syndrome is the careful characterization and diagnosis of the disease, which includes clinical, cytogenetic, biological and molecular investigation of individual patients. Today therapeutic approaches to the treatment of such patients are differentiated and depend, first of all, on the subtype of the disease, age, general condition of the patients and the possibility of allogeneic hematopoietic cell transplantation. For young patients, the best option is transplantation, whereas in older patients, the standard of therapy is the use of hypomethylating agents (azacitidine, decitabine). These drugs promote hematologic improvement, elimination of transfusion dependence and prolongation of the duration of both general and leukemia free survival in elderly patients with concomitant pathology.

Despite the fact that therapy with hypomethylating drugs is widely used and has good results, many respondents are losing their response within 1-2 years. Reasons for the development of resistance to this type of treatment are still unclear, and the insensitivity to drugs is associated with very poor prognosis in patients with all subtypes of myelodysplastic syndrome. Such data and the presence of numerous genetic and epigenetic mechanisms for the development of this pathology have prompted the use of combinations of drugs with different application points and are relevant in terms of research. In the literature review, the results of clinical studies on the use of hypomethylating agents in patients with MDS of low and high risk, as in monotherapy and combined schemes are presented.

The nearest prospect of treatment of myelodysplastic syndrome is the creation of new treatment regimens based on a combination of drugs of different pathogenetic direction for the elimination of the dysplastic clone in order to achieve not only long-term remissions, but also lengthening the duration of overall survival, especially for patients with high risk myelodysplastic syndrome.

Keywords: myelodysplastic syndrome, decitabine, azacitidine, overall survival, leukemia free survival, acute leukemia.
\end{abstract}

\section{Introduction}

Despite the long history of seeking therapeutic approaches to treatment of myelodysplastic syndrome (MDS), many aspects of this problem have not been resolved, some of them contradictory and, unfortunately, at present, the complete cure of this pathology for most patients remains impossible not only in Ukraine, but also in developed countries of the world. Scientists are convinced that the only radical method of treating this disease, for today, is the allogeneic hematopoietic cell transplantation (HTC). However, the problem with the presence an HLA- compatible donor makes this procedure far from being a routine practice and can only be used in $10 \%$ of patients.

As noted at the 57th Congress of ASH, over the past 10 years, considerable progress has been made in the study of the pathogenesis of MDS, however, no significant progress has been made in the therapeutic approaches of individual MDS subtypes. Until recently, only substitution therapy used in such patients was hemotransfusion, which for some time improves the well-being of the patient, however, due to the development of transfusion dependence with clinical manifestations of iron overload, it is become the most common cause of non-leukemia mortality $[1,2]$. It is proved that increasing the level of ferritin at each $500 \mathrm{ng} / \mathrm{ml}$ increases mortality by $36 \%$ [3].

With the advent of new drugs, the view on the problem of treatment of MDS has changed. In modern haematology therapeutic approaches in such patients are differentiated and depend on the age of patients, general condition, the presence of concomitant pathology and the possibility of alloHTC. So in patients with low risk MDS, the main goal of therapy is to reduce the number of blood transfusion and improve the quality of life of the patient $[4,5]$. Instead, with the more aggressive subtypes of MDS, the end point is to reduce the risk of transformation into acute myeloid leukaemia (AML) and to lengthen the duration of overall survival [6]. 
In order to find the "golden standard" for MDS treatment was urgent the study of the effectiveness of pathogenetic-directed therapy with the help of hypomethylating agents (decitabine (DAC), azacitidine (AZA)), registered by the Ministry of Food and Drug Administration, USA (FDA ) for clinical use in 2004 and 2006, respectively.

By studying the pathogenetic mechanisms of the formation of MDS, an increase in the methylation of the p15IN4B gene encoding the protein-inhibitor of cyclin-dependent kinases 4 and 6 in this pathology was detected [7]. Hypermethylating of this gene was observed in about $50 \%$ of cases, with a higher incidence in patients with subtypes of the refractory anaemia with excess blasts (RAEB) $[8,9]$. Inactivation of DNA-methyltransferases leads to the restoration of the imbalance of silent genes and impaired cell differentiation in this nosology [10].

\section{Aim of the research}

Obtain new information on the use of hypomethylating agents (decitabine and azacitidine) in treating patients with different degrees of myelodysplastic syndrome according to IPSS.

\section{Materials and methods}

The article analyzes 41 scientific publications for the period 2012-2017, in which information on the effectiveness of hypomethylating agents in patients with myelodysplastic syndrome is presented.

\section{Result}

Azacitidine in the treatment of low risk and high risk MDS according to IPSS. According to NCCNV2.2017 recommendations [11] for patients with MDS low and intermediate-1 risk, according to IPSS, this therapy is used:

- patients with endogenous erythropoietin (EPO) less than $500 \mathrm{mU} / \mathrm{ml}$ in the ineffectiveness of therapy with recombinant human erythropoietin ( $\mathrm{rHu}$ Epo) in monotherapy or in combination with granulocyte colony-stimulating factor [12];

- patients with an EPO level above $500 \mathrm{mU} / \mathrm{ml}$ in the presence of adverse signs of response to immunosuppressive therapy with cyclosporine or antithymocyte globulin (ATG);

- patients with clinically significant thrombocytopenia and/or neutropenia.

It has been shown that hypomethylating agents, in particular AZA, prolong the survival of patients, regardless of age, FAB or WHO subtype of the disease, the percentage of blast and karyotype (although some researchers have noted a better result in patients with the presence of -7/del7q) [13], significantly lower the risk of transformation in the AML, and not only reduce the transfusion dependence of patients, but also prolong the duration of the response in the previously treated patients $[14,15]$.

According to the NCCN standards for high risk MDS patients (intermediate-2/high IPSSscale risk), intensive chemotherapy followed by HTC is a priority treatment. However, in the absence of a donor or the impossibility of conducting this procedure (late age of patients, the presence of concomitant pathology) by the standard of treatment until recently was the use of hypomethylating agents, as the first line of therapy in this cohort of patients [11, 16]. In connection with the expansion of knowledge about their mechanism of action and side effects, indications for the use of this group of drugs have become wider today. Thus, a group of British researchers led by Craddock believe that AZA may be the drug of choice for the treatment of recurrences after an unsuccessful transplant, resulting in not only a satisfactory response in the fourth part of the treatment $(15 \%$ confirmed complete remission), but also lengthening the duration of 2 years of survival until $48 \%$ vs. $12 \%$ for the general population $[17,18]$. No less interesting is the study on the use of AZA as a supportive therapy after a transplant. After 4-courses AZA at a dose of $32 \mathrm{mg} / \mathrm{m}^{2}$ for 5 days, an extension of the duration of not only general, but also leukaemia free survival in these patients was noted [19, 20]. A group of researchers from Lebanon in 2014 not only confirmed the above results, but also found that AZA is more effective in the presence of a normal diploid karyotype, which in some way denies other literature on good performance in patients with chromosome 7 monosomy. 
Despite the contradictions in the effectiveness of the results azacitidine with an abnormal karyotype, the question remains interesting and requires a deeper analysis.

Satisfactory results of therapy with hypomethylating agents have become the impetus for further study of their effectiveness in patients with MDS of various prognostic subtypes. In 2011, phase III data from the randomized trial of CALGB-9221 (Cancer and Leukemia Group B), subcutaneous administration of AZA ( $75 \mathrm{mg} / \mathrm{m}^{2} /$ day, 1-7 days, every 28 days, $\left.\mathrm{n}=99\right)$, were reported against the best supportive care (BSC) $(n=92)$ in patients of all MDS subtypes according to the FAB classification, which showed significantly higher rates of response to treatment AZA $60 \%$ vs. $5 \%$ with traditional therapy $(\mathrm{p}<0.001)$. The time to progression or death was statistically longer in the azacitidine group compared to the comparative branch: 21 months against 13 months, accordinly $(\mathrm{p}<0.007)$. The number of treated, who progressed was significantly higher in the comparison group of $38 \%$ versus $15 \%$, respectively $(\mathrm{p}<0.001)$. The median time to reaching the best response was 64 and 93 days with AZA and BSC, respectively. However, no significant difference was found in the overall survival time of these patients, 20 months against 14 months $(p=0.1)$, since according to the protocol after 4 months treatment with the best concomitant therapy patients received further treatment with azacitidine. In all of the subjects receiving the hypomethylating agent, the quality of life improved, fatigue, shortness of breath were reduced and physical activity increased [11, 21].

No less interesting are the results of the phase III, international, multicenter, randomized, AZA-001 trial with subcutaneous administration of AZA in dose $75 \mathrm{mg} / \mathrm{m}^{2} /$ day for $1-7$ days in a 28-day cycle in 179 MDS intermediate-2/high risk patients versus conventional care regimens (CCR) that included substitution therapy (105 patients), low-dose AraC (49 patients) and intensive chemotherapy (25 patients). Not only the higher percentage of the hematologic response $(\mathrm{p}=0.0001)$ was established according to the IWG 2000 criteria, but also the longer duration of the overall (24.5 months versus 15 months, $\mathrm{p}=0.0001)$ and the two-year survival $(50.8 \%$ vs. $26.2 \%, \mathrm{p}<0.0001)$ with azacitidine versus induction/consolidation therapy. The duration of the response in the azacitidine branch was also significantly longer than 13.6 months against 5.2 months in the comparison group $(\mathrm{p}=0.0002)$. AZA significantly increased the time to transformation in AML (18.7 months versus 11.5 months, $\mathrm{p}<0.001$ ) and decreased dependence on transfusion in $45 \%$ of transfusion dependent patients versus $11.4 \%$ in group comparison. Interesting the fact that the hypomethylating agent was significantly more effective in patients with unfavorable cytogenetic aberrations, in particular at $-7 /$ del (7q), in which the duration was significantly longer than the overall survival (13.1 months vs. 4.6 months, $\mathrm{p}=0.00001$ ) and leukemia free survival (17.8 months vs. 11.5 months, $\mathrm{p}<0.0001)$ [22].

The satisfactory response to AZA therapy has led to the study of alternative doses and treatment regimens for patients.

$-75 \mathrm{mg} / \mathrm{m}^{2} /$ day subcutaneously according to the scheme " $5-2-2$ " every 28 days;

$-50 \mathrm{mg} / \mathrm{m}^{2} /$ day subcutaneously according to the scheme " $5-2-5$ " every 28 days;

$-75 \mathrm{mg} / \mathrm{m}^{2} /$ day subcutaneously for 5 days every 28 days.

When comparing the results of 5 days the hematological improvement was achieved in $44 \%, 45 \%$ and $56 \%$ of cases with AZA 5-2-2, AZA 5-2-5, and AZA-5, respectively. All three alternative regimes provided the development of independence from transfusion in $50 \%, 55 \%$ and $64 \%$ of treated patients, respectively. The percentage of complications was less pronounced at the 5 -day regimen of $75 \mathrm{mg} / \mathrm{m}^{2} /$ day, which was $58 \%$ compared with $84 \%$ under the scheme $5-2-2$ and $77 \% 5-2-5$, This scheme may be recommended for use in ambulatory conditions [23, 24].

At this stage, the II-III phase of the study, the tablet form of azacitidine CC-486, focuses on patients with MDS (both primary and pre-treated), with chronic myelomonocytic leukemia and AML [25].

The design of the study was as follows (Fig. 1).

In 2015, the first results of I phase of the study are published. It was found that the study drug has high stability, good bioavailability and a longer half-life than the subcutaneous administration of the drug. Combined mode contributed to a better response of $73 \%$ versus $42 \%$ when using only the tablet form. Among the major complications most often encountered during therapy, except for febrile neutropenia were gastrointestinal disorders [25]. 


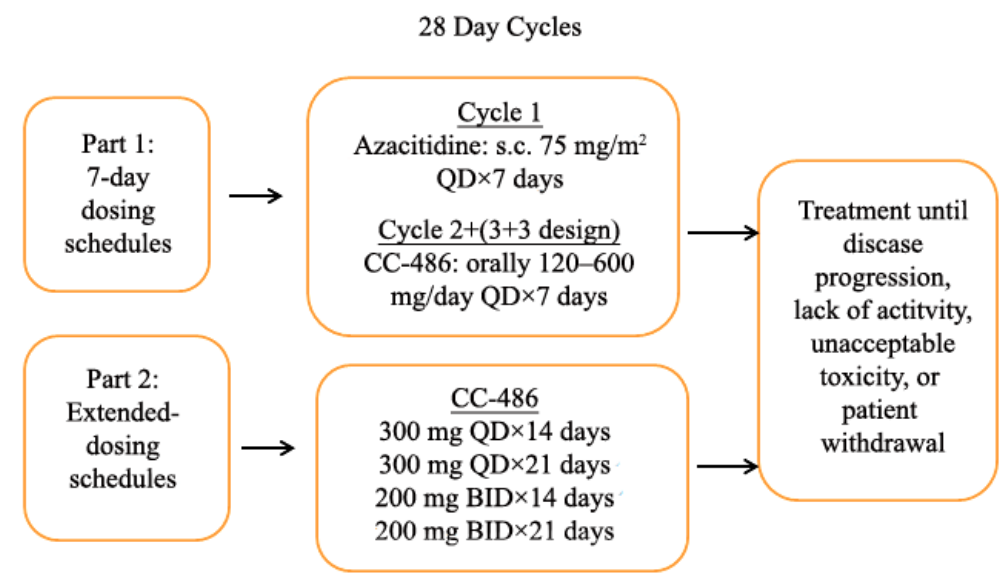

Fig. 1. AZAPHUS 2007 CL005, phase 1 study of the azacitidine tablet form (CC-486) in patients with MDS, CMML, and AML

In connection with the revealed pharmacokinetic properties of SS-486, researchers believe that prolonged use of the drug may be effective in patients with thrombocytopenia or transfusion dependent anaemia, which is confirmed by the clinical study of the III phase of the QUAZAR low risk MDS protocol AZA-MDS-003 [26] or prolong the response in the period after transplantation - I/II phase of the study CC-486-AML-002 [27].

Decitabine in low and high risk MDS therapy. Decitabine (DAC), which appeared in the Ukrainian market in 2008, is also effective in treating MDS.

Dosage questions and DAC injection modes are investigated on a permanent basis. Described in the literature, the efficacy of maximum single dose doses of $300-500 \mathrm{mg} / \mathrm{m}^{2}$ and modes of long 72-hour infusion of small doses (total dose $125-225 \mathrm{mg} / \mathrm{m}^{2}$ ) at intervals of 6 weeks did not meet the expectations of scientists, because such therapy was accompanied by pronounced cytopenic states, the severity of which was higher than the result obtained, in this connection, the schemes are practically not used in modern practice.

North American researchers presented the results of a multicenter phase III study to evaluate the effectiveness of DAC $\left(15 \mathrm{mg} / \mathrm{m}^{2} / \mathrm{h}\right.$ for 3 hours every 8 hours $1-3$ days every 6 weeks) compared with the best concomitant therapy in elderly patients with primary or secondary MDS. The use of this regimen did not show a significant difference in the duration of overall survival of patients compared with the control group, however, it prolonged the median time before transformation or death (12 months versus 6.8 months, $\mathrm{p}=0.03)$. The response rate for treatment was fairly low and reached 17 [11].

In 2005, Kantarjian et al presented the results of the Anderson Cancer Cente study, which evaluated the effectiveness of DAC in three regimens: $10 \mathrm{mg} / \mathrm{m}^{2}$ intravenous 10 days, $20 \mathrm{mg} / \mathrm{m}^{2}$ 5 days and $20 \mathrm{mg} / \mathrm{m}^{2}$ subcutaneous 5 days at intervals between courses 4 weeks. The complete remission of the 5-day regimen in the form of proven infusion was the highest and was $39 \%$ versus $21 \%$ for subcutaneous administration and $24 \%$ for the 10 -day regimen, whereby the 5-day intravenous regimen was considered to be the most optimal for use [28].

Instead, newer studies comparing 3-day treatment with $15 \mathrm{mg} / \mathrm{m}^{2}$ three times a day and a 5 -day regimen at $20 \mathrm{mg} / \mathrm{m}^{2} /$ day showed a higher percentage of overall response rate when using the 3-day regimen ( $29.4 \%$ vs. $25,5 \%$ in the comparison group), however, no difference was found in the hematological improvement and the leukemia free survival. Side effects associated with myelosuppression $(85.6 \%)$ and infections (43.2 \%) were also not significantly different [29, 30].

Regardless, a group of German researchers analyzed the 5-day regimen of using $20 \mathrm{mg} / \mathrm{m}^{2}$ doses of decibitin IV as compared with concomitant therapy in older patients with intermediate or high risk MDS versus indications for intensive chemotherapy. Median survival without progres- 
sion was significantly longer in patients treated with decitabine 6.6 months against 3.0 months $(\mathrm{p}=0.004)$, and the risk of transformation during the year in these patients was significantly lower ( $22 \%$ vs. $33 \%, p=0.036$ ). At the same time, the two study groups did not find a significant difference in the duration of overall survival ( 10.1 months versus 8.5 months, $\mathrm{p}=0.38)$ and the leukemia free survival ( 8.8 months versus 6.1 months, $\mathrm{p}=0.38$ ). In the DAC branch, complete and partial remission was observed at $13 \%$ and $6 \%$, respectively, with additional hematologic improvement in $15 \%$ of patients relative to the IWG 2006 criteria, with a $2 \%$ hematological improvement in the adjunctive therapy line with no hematologic response [21].

Differentiated efficacy of azacitidine and decitabine in the treatment of MDS. In 2013 was published a study that aimed to directly compare the efficacy and safety of azacytidine and decitabine Among the 300 treated patients, of whom 203 received azacitidine and 97 decitabine, no significant difference was found in the overall response ( $44 \%$ vs. $52 \%$ ), overall survival ( 26 months versus 22.9 months) and survival rate of leukemia ( 7.7 vs. 7.0 months), and in particular in the number of transformed patients per year (16\% vs. $22 \%)$. It was interesting to note the fact that the age-related survival rate was significant: in patients older than 65 years, the survival rate was significantly longer in the azatsitydine group (HR 3; $95 \%$ CI 1.22-7.37; $\mathrm{p}=0.017$ ), with the same difference in the group of subjects under 65 years old not statistically significant (HR 1.17; $95 \%$ CI $0.54-2.54 ; \mathrm{p}=0.7$ ). Instead, the development of severe cytopenia and infectious complications was significantly higher in the group treated with decitabine [31].

Interesting and rather contradictory are the results of the phase II study of the safety and efficacy of the use of small doses of decitabine in comparison with low doses of azacytidine in a group of low-risk MDS patients receiving intravenous/subcutaneously azacitidine $75 \mathrm{mg} / \mathrm{m}^{2}$ daily or $20 \mathrm{mg} / \mathrm{m}^{2}$ of decitabine daily intravenously 3 consecutive days in a 28-day cycle. It was found that the percentage of patients who responded to treatment ( $70 \%$ vs. $49 \%, \mathrm{p}=0.03)$ and those who observed the development of transfusional independence ( $32 \%$ vs. $16 \%$, p=0.02) was significantly higher when using decitabine. The cytogenetic response rates were $61 \%$ and $25 \%(\mathrm{p}=0.02)$, respectively, including the duration of leukemia free survival which was longer in the decitabine 20 months versus 13 months for azacitidine [32].

Recent publications on the MDS Clinical Research Consortium have demonstrated a superior response in female patients with decitabine compared with azacitidine, but with a longer survival in patients treated with azacitidine ( 21.1 months versus 13.2 months, $p=0.0014$ ). The reasons for this difference, according to the authors, may be differences in the pharmacokinetics of drugs in women and men [33]. Other studies show the dependence of the response to treatment with hypomethylating agents on peripheral blood parameters. In particular, the platelet count $\geq 100 \times 10^{9} / 1(\mathrm{p}=0.007)$ and the leukocyte count $<3.0 \times 10^{9} / 1(\mathrm{p}=0.03)$ are considered to be favorable predictors of the likelihood of a satisfactory response to therapy. Additionally, it has been established that the lack of cytogenetic changes or the presence of favorable cytogenetic aberrations significantly increases the likelihood of a satisfactory response to treatment [34]. Instead, Bejaretal found that the presence of TET2 and DNMT3A mutations doubly increases the likelihood of receiving response to therapy [35]. However, the mutation status is not associated with the duration of the response received or the improvement of survival in any of the studies.

The presence of numerous genetic and epigenetic mechanisms underlying MDS has led to the use of combinations of drugs with different application points. Thus, with a combination of lenalidomide and azacitidine, the response to treatment is increased to $69 \%$ versus $56 \%$ when monotherapy azacitidine is used in patients with high risk MDS. Instead, such a combination is not associated with prolonged leukaemia free survival where its duration is 18.8 months against 33.2 months with monotherapy [36].

At the study stage, a combination of azacitidine with etanercept or vorinostat in patients with high risk MDS, which showed a high response rate of $72 \%$ for the first combination and $82 \%$ for another [37], a combination of histone deacetylase inhibitors (panobinostat) and azacitidine also showed a higher percentage of response, $27.5 \%$ vs. $14.3 \%$, no difference was found in the duration 
of overall survival (70\% vs. $60 \%$ in mono-regimens), and the percentage of complications was significantly higher in the combined regimen [38].

No less striking were the results (phase II) of the use of the azacitidine scheme with the monoclonal antibody, gemtuzumab, in combination with hydroxyurea. Accordingly, response was received in $70 \%$ of the patients with high risk MDS. The overall survival time for these patients was 10 months, and the response achieved was at least 8 months. [39].

Despite the fact that hypomethylating agents significantly improve the treatment outcome in patients with MDS, only half of the patients receive an objective response, and most respondents lose it within 1-2 years. The genesis of primary and secondary resistance to hypomethylating therapy is not established, and the failure of therapy is associated with a very poor prognosis in MDS patients of both low and high risk of transformation in the AML [40]. The overall survival rate in patients with high risk MDS after failure of therapy with azacitidine or decitabine is 5.6 and 4.3 months respectively [41]. In a report by the Moffitt group, the overall survival time after ineffectiveness of azacitidine, analyzed in 280 patients with low-risk MDS, was 18.5 months $(95 \%$ confidence interval, 13.5-23.5 months), and decreased in patients with intermediate risk MDS according to IPSS down to 15 months [14]. Another retrospective analysis of 59 patients with IPSS low risk MDS with primary or secondary azacitidine ineffectiveness showed similarly low overall survival rates in these patients -16.7 months. [37]. The median of the overall survival of 74 patients with secondary AL after failure of azacitidine was even lower and was 3.4 months with a probability of 1 year survival of only $8 \%$ [41].

\section{Discussion}

After analyzing the effectiveness of the use of hypomethylating mediators in patients with MDS, we can talk about a significant progress in the treatment of this pathology after the introduction of pathogenetic therapy, and in particular, the hypomethylating drugs. In view of the impossibility of fully curing these patients because of the limitations in the allogeneic hematopoietic stem cells transplantation, the use of drugs of this subgroup, according to most authors, not only improves the quality of life, but significantly lengthens the duration of both overall and leukaemia free survival, in particular in patients with high risk MDS.

Expansion of indications for the use of these drugs in patients after transplantation in order to lengthen the duration of remission achieved, looks like another promising direction of therapeutic tactics for this group of patients.

Although most authors point to a relatively high incidence of complications that develop with the use of hypomethylating agents, the constant process of hypermethylation of DNA in the MDS necessitates their continued use until the progression of the disease or unacceptable toxicity.

The development of resistance to this type of treatment contributed to the further search for new schemes based on a combination of drugs of different pathogenetic orientation and it is the nearest prospect of the treatment of MDS of various FAB subtypes.

\section{Conclusion}

1. An analysis of literature sources has shown higher efficacy of hypomethylating agents compared to the best supportive care or other standard treatment regimens in low and high risk MDS patients.

2. The absence of significant differences in the overall survival and leukemia free survival, the percentage of response and reduction of transfusion dependence, as well as the number of transformed patients during the year when using azacitidine and decitabine, suggests the use of both hypomethylating agents for the treatment of all subtypes of MDS.

3. In low-risk and high-risk patients with MDS over 65 years of age a drug of choice is azacitidine, since when used in this cohort, respondents have significantly higher survival rates and significantly lower incidence of severe cytopenia and infectious complications compared with the use of decitabine. In younger patients, this trend was statistically insignificant and the drug of choice for them may be as azacitidine and decitabine. 
4. The nearest prospect of treatment of myelodysplastic syndrome is the creation of new treatment regimens based on a combination of drugs of different pathogenetic direction.

\section{References}

[1] Cogle, C. R., Reddy, S. R., Chang, E., Papoyan, E., Broder, M. S., McGuire, M., Binder, G. (2017). Early treatment initiation in lower-risk myelodysplastic syndromes produces an earlier and higher rate of transfusion independence. Leukemia Research, 60, 123-128. doi: 10.1016/j.leukres.2017.07.008

[2] Malcovati, L. (2007). Impact of transfusion dependency and secondary iron overload on the survival of patients with myelodysplastic syndromes. Leukemia Research, 31, 2-6. doi: 10.1016/s01452126(07)70459-9

[3] Angelucci, E., Urru, S. A. M., Pilo, F., Piperno, A. (2017). Myelodysplastic Syndromes And Iron Chelation Therapy. Mediterranean Journal of Hematology and Infectious Diseases, 9 (1), e2017021. doi: 10.4084/mjhid.2017.021

[4] Fenaux, P., Ades, L. (2013). How we treat lower-risk myelodysplastic syndromes. Blood, 121 (21), 4280-4286. doi: 10.1182/blood-2013-02-453068

[5] Malcovati, L., Hellstrom-Lindberg, E., Bowen, D., Ades, L., Cermak, J., del Canizo, C. et. al. (2013). Diagnosis and treatment of primary myelodysplastic syndromes in adults: recommendations from the European LeukemiaNet. Blood, 122 (17), 2943-2964. doi: 10.1182/blood-2013-03-492884

[6] Sekeres, M. A., Cutler, C. (2013). How we treat higher-risk myelodysplastic syndromes. Blood, 123 (6), 829-836. doi: 10.1182/blood-2013-08-496935

[7] Visconte, V., Selleri, C., Maciejewski, J. P., Tiu, R. V. (2014). Molecular Pathogenesis of Myelodysplastic Syndromes. Translational Medicine UniSa, 8 (4), 19-30.

[8] Kulasekararaj, A. G., Jiang, J., Smith, A. E., Mohamedali, A. M., Mian, S., Gandhi, S. et. al. (2014). Somatic mutations identify a subgroup of aplastic anemia patients who progress to myelodysplastic syndrome. Blood, 124 (17), 2698-2704. doi: 10.1182/blood-2014-05-574889

[9] Harada, H., Harada, Y. (2015). Recent advances in myelodysplastic syndromes: Molecular pathogenesis and its implications for targeted therapies. Cancer Science, 106 (4), 329-336. doi: 10.1111/cas.12614

[10] Duchmann, M., Itzykson, R. (2017). Choosing a hypomethylating agent in MDS: does gender matter? Leukemia \& Lymphoma, 58 (6), 1277-1278. doi: 10.1080/10428194.2017.1283035

[11] Greenberg, P. L., Stone, R. M., Al-Kali, A., Barta, S. K., Bejar, R., Bennett, J. M. et al. (2017). Myelodysplastic Syndromes, Version 2.2017, NCCN Clinical Practice Guidelines in Oncology. Journal of the National Comprehensive Cancer Network, 15 (1), 60-87. doi: 10.6004/jnccn.2017.0007

[12] Mies, A., Platzbecker, U. (2017). Increasing the effectiveness of hematopoiesis in myelodysplastic syndromes: erythropoiesis-stimulating agents and transforming growth factor- $\beta$ superfamily inhibitors. Seminars in Hematology, 54 (3), 141-146. doi: 10.1053/j.seminhematol.2017.06.004

[13] Schanz, J., Braulke, F., Mufti,G. J., Crisa, E., Kulasekararaj, A., Nachtkamp, K. et. al. (2014). Clinical Characteristics and Treatment Allocations in Patients with Myelodysplastic Syndromes and Monosomy 7: Results from an International Multicenter Study Suggest That Hypomethylating Agents Significantly Improve Overall- and AML-Free Survival in Patients Classified As Very High Risk By IPSS-R. Blood, 124 (21), 4656.

[14] Apuri, S., Al Ali, N., Padron, E., Lancet, J. E., List, A. F., Komrokji, R. S. (2017). Evidence for Selective Benefit of Sequential Treatment With Hypomethylating Agents in Patients With Myelodysplastic Syndrome. Clinical Lymphoma Myeloma and Leukemia, 17 (4), 211-214. doi: 10.1016/j.clml.2016.10.003

[15] Madanat, Y., Sekeres, M. A. (2017). Optimizing the use of hypomethylating agents in myelodysplastic syndromes: Selecting the candidate, predicting the response, and enhancing the activity. Seminars in Hematology, 54 (3), 147-153. doi: 10.1053/j.seminhematol.2017.06.001

[16] Timothy, A., Razavi, G. E. (2016). Targeted Therapy in Myelodysplastic Syndrome. ECronicon Cancer, 2.1, 34-44.

[17] Tessoulin, B., Delaunay, J., Chevallier, P., Loirat, M., Ayari, S., Peterlin, P. et. al. (2014). Azacitidine salvage therapy for relapse of myeloid malignancies following allogeneic hematopoietic SCT. Bone Marrow Transplantation, 49 (4), 567-571. doi: 10.1038/bmt.2013.233 
[18] Craddock, C., Labopin, M., Robin, M., Finke, J., Chevallier, P., Yakoub-Agha, I. et. al. (2016). Clinical activity of azacitidine in patients who relapse after allogeneic stem cell transplantation for acute myeloid leukemia. Haematologica, 101 (7), 879-883. doi: 10.3324/haematol.2015.140996

[19] De Lima, M., Giralt, S., Thall, P. F., de Padua Silva, L., Jones, R. B., Komanduri, K. et. al. (2010). Maintenance therapy with low-dose azacitidine after allogeneic hematopoietic stem cell transplantation for recurrent acute myelogenous leukemia or myelodysplastic syndrome. Cancer, 116 (23), 5420-5431. doi: $10.1002 /$ cncr. 25500

[20] Antar, A., Kharfan-Dabaja, M. A., Ghaddara, H. A., Mahfouz, R., Bazarbach, A. (2014). Azacitidine Maintenance after Allogeneic Stem Cell Transplantation Is Feasible in Patients with Acute Myeloid Leukemia and Myelodysplasia. Blood, 124 (21), 5884.

[21] Lubbert, M., Suciu, S., Baila, L., Rüter, B. H., Platzbecker, U., Giagounidis, A. et. al. (2011). Low-Dose Decitabine Versus Best Supportive Care in Elderly Patients With Intermediate- or High-Risk Myelodysplastic Syndrome (MDS) Ineligible for Intensive Chemotherapy: Final Results of the Randomized Phase III Study of the European Organisation for Research and Treatment of Cancer Leukemia Group and the German MDS Study Group. Journal of Clinical Oncology, 29 (15), 1987-1996. doi: 10.1200/ jco.2010.30.9245

[22] Gore, S. D., Fenaux, P., Santini, V., Bennett, J. M., Silverman, L. R., Seymour, J. F. et al. (2013). A multivariate analysis of the relationship between response and survival among patients with higher-risk myelodysplastic syndromes treated within azacitidine or conventional care regimens in the randomized AZA-001 trial. Haematologica, 98 (7), 1067-1072. doi: 10.3324/haematol.2012.074831

[23] Musto, P., Maurillo, L., Spagnoli, A., Gozzini, A., Rivellini, F. et. al. (2010). Azacitidine for the treatment of lower risk myelodysplastic syndromes. Cancer, 116 (6), 1485-1494. doi: 10.1002/ cncr.24894

[24] Garcia-Delgado, R., de Miguel, D., Bailen, A., Gonzalez, J. R., Bargay, J., Falantes, J. F. et. al. (2014). Effectiveness and safety of different azacitidine dosage regimens in patients with myelodysplastic syndromes or acute myeloid leukemia. Leukemia Research, 38 (7), 744-750. doi: 10.1016/ j.leukres.2014.03.004

[25] Cogle, C. R., Scott, B. L., Boyd, T., Garcia-Manero, G. (2015). Oral Azacitidine (CC-486) for the Treatment of Myelodysplastic Syndromes and Acute Myeloid Leukemia. The Oncologist, 20 (12), 1404-1412. doi: 10.1634/theoncologist.2015-0165

[26] The efficacy and safety of oral azacitidine plus best supportive care versus placebo and best supportive care in subjects with red blood cell (RBC) transfusion-dependent anemia and thrombocytopenia due to International Prognostic Scoring System (IPSS) low risk myelodysplastic syndrome(MDS) (2012). ClinicalTrials. Available at: http://www.clinicaltrials.gov/ct2/show/NCT01566695 Last accessed: 12.01.2015

[27] Safety Study of Oral Azacitidine (CC-486) as Maintenance Therapy After Allogeneic Hematopoietic Stem Cell Transplantation in Subjects With Acute Myeloid Leukemia or Myelodysplastic Syndromes (2013). ClinicalTrials. Available at: https://clinicaltrials.gov/ct2/show/NCT01835587

[28] Santini, V. (2012). Novel therapeutic strategies: hypomethylating agents and beyond. Hematology. American Society of Hematology. Education Program, 2012 (1), 65-73. doi: 10.1182/asheducation-2012.1.65

[29] Killick, S. B., Carter, C., Culligan, D., Dalley, C., Das-Gupta, E. et. al. (2013). Guidelines for the diagnosis and management of adult myelodysplastic syndromes. British Journal of Haematology, 164 (4), 503-525. doi: 10.1111/bjh.12694

[30] Wu, D., Du, X., Jin, J., Xiao, Z., Shen, Z., Shao, Z. et. al. (2015). Decitabine for Treatment of Myelodysplastic Syndromes in Chinese Patients: An Open-Label, Phase-3b Study. Advances in Therapy, 32 (11), 1140-1159. doi: 10.1007/s12325-015-0263-8

[31] Lee, Y.-G., Kim, I., Yoon, S.-S., Park, S., Cheong, J. W. et. al. (2013). Comparative analysis between azacitidine and decitabine for the treatment of myelodysplastic syndromes. British Journal of Haematology, 161 (3), 339-347. doi: 10.1111/bjh.12256

[32] Jabbour, E., Short, N. J., Montalban-Bravo, G., Huang, X., Bueso-Ramos, C., Qiao, W. et. al. (2017). Randomized phase 2 study of low-dose decitabine vs low-dose azacitidine in lower-risk MDS and MDS/MPN. Blood, 130 (13), 1514-1522. doi: 10.1182/blood-2017-06-788497 
[33] DeZern, A. E., Zeidan, A. M., Barnard, J., Hand, W., Al Ali, N., Brown, F. et. al. (2016). Differential response to hypomethylating agents based on sex: a report on behalf of the MDS Clinical Research Consortium (MDS CRC). Leukemia \& Lymphoma, 58 (6), 1325-1331. doi: 10.1080/ 10428194.2016.1246726

[34] Traina, F., Visconte, V., Elson, P., Tabarroki, A., Jankowska, A. M., Hasrouni, E. et. al. (2013). Impact of molecular mutations on treatment response to DNMT inhibitors in myelodysplasia and related neoplasms. Leukemia, 28 (1), 78-87. doi: 10.1038/leu.2013.269

[35] Bejar, R., Lord, A., Stevenson, K., Bar-Natan, M., Perez-Ladaga, A., Zaneveld, J. et. al. (2014). TET2 mutations predict response to hypomethylating agents in myelodysplastic syndrome patients. Blood, 124 (17), 2705-2712. doi: 10.1182/blood-2014-06-582809

[36] Komrokji, R. S., Sekeres, M. A., Barnard, J., Barnard, J., Alali, N., DeZern, A. E. et. al. (2016). Optimal treatment order of lenalidomide and hypomethylating agents for lower-risk myelodysplastic syndromes: a report on behalf of the MDS Clinical Research Consortium. Blood, 128 (22), 4322.

[37] Prebet, T., Thepot, S., Gore, S. D., Dreyfus, F., Fenaux, P., Vey, N. (2012). Outcome of patients with low-risk myelodysplasia after azacitidine treatment failure. Haematologica, 98 (2), 18-19. doi: 10.3324/ haematol.2012.071050

[38] Garsia-Manero, G., Sekeres, M. A., Edyed, M., Alimena, G., Graux, C., Cavenagh, J. D. et. al. (2015). Panobinostat plus azacitidine in adult patients with MDS, CMML or AML: results of a phase 2b study. Blood, 126 (3), 2861.

[39] Zeidan, A. M., Kharfan-Dabaja, M. A., Komrokji, R. S. (2014). Beyond hypomethylating agents failure in patients with myelodysplastic syndromes. Current Opinion in Hematology, 21 (2), 123-130. doi: 10.1097/moh.0000000000000016

[40] Duong, V. H., Lin, K., Reljic, T., Kumar, A., Al Ali, N. H., Lancet, J. E. et. al. (2013). Poor Outcome of Patients With Myelodysplastic Syndrome After Azacitidine Treatment Failure. Clinical Lymphoma Myeloma and Leukemia, 13 (6), 711-715. doi: 10.1016/j.clm1.2013.07.007

[41] Prebet, T., Gore, S. D., Thepot, S., Esterni, B., Quesnel, B., Beyne Rauzy, O. et. al (2012). Outcome of acute myeloid leukaemia following myelodysplastic syndrome after azacitidine treatment failure. British Journal of Haematology, 157 (6), 764-766. doi: 10.1111/j.1365-2141.2012.09076.x 